\title{
Subacute combined degeneration of spinal cord, optic atrophy and thrombocytopenia without anemia secondary to B12 deficiency
}

\author{
Perera $C^{1}$, Sivagnanam F.G. ${ }^{2}$, Ranaweera Y. ${ }^{3}$, Wijesooriya S. ${ }^{4}$
}

\begin{abstract}
Long-standing vitamin B12 deficiency can lead to neurological manifestations such as optic atrophy and sub acute combined degeneration of the cord without causing significant anemia. In addition, folic acid supplementation could mask the hematological manifestations of vitamin B12 deficiency and delay the presentation of neurological manifestations

We present a case of a 32-year-old vegan woman on long-term sodium valproate and folic acid treatment presenting with progressive weakness of bilateral lower limbs for three months and reduced vision in both eyes for two weeks duration. Further evaluation revealed optic atrophy, combined degeneration of the spinal cord, macrocytosis, thrombocytopenia, and severely deficient serum B12 levels. With B12 replacement therapy, the patient's overall wellbeing improved.
\end{abstract}

Keywords : Vitamin B12, subacute combined degeneration, thrombocytopenia, folic acid supplementation.

\section{Introduction}

B12 deficiency is known to cause neurological and psychiatric manifestations. ${ }^{1}$ The classic neurological finding in B12 deficiency is described as subacute combined degeneration of dorsal and lateral columns of the spinal cord. ${ }^{1}$. In addition, it is associated with optic atrophy, peripheral neuropathy, cognitive changes, and affective disorders. ${ }^{1}$

Neuropsychiatric manifestations of B12 deficiency are identified in the absence of anemia or macrocytosis ${ }^{2}$. Administration of folic acid to a patient with underlying B12 deficiency may potentially mask the hematological manifestations and may worsen the neurological manifestations. $^{3}$

\section{Case Report}

Our patient was a 32-year-old unmarried woman who has been working in a garment factory. She had been taking sodium valproate $200 \mathrm{mg}$ three times a day and folic acid $5 \mathrm{mg}$ daily since 11 years of age for epilepsy. She has had several episodes of seizures when she

${ }^{1}$ The National Hospital, Sri Lanka (NHSL), Sri Lanka

2,3 Consultant Physician, NHSL, Sri Lanka

${ }^{4}$ Senior registrar in Medicine NHSL,Sri Lanka

Corresponding Author:

Charith Perera; chulaguptha@gmail.com

(iD) https://orcid.org/0000-0002-8431-4685 missed the doses.She had stiffness and mild weakness of the right upper limb since childhood. She has not consumed animal-based food such as fish, meat, and dairy products for the last 10 years.

She started feeling lethargic 4 months ago. She noted pins and needles in her both feet which started 3 months ago. Then she has experienced progressive worsening of difficulty in walking which stopped her from going to work. She did not have bladder or bowel disturbances. She denied back pain. There had not been any trauma or injury to the spine. She also experienced a significant weight loss over the last 6 months. She did not have a fever or contact history of tuberculosis. She did not have diarrhea or abdominal pain to suggest malabsorption, and other clinical evidence of autoimmune conditions to suggest pernicious anemia such as vitiligo and hypothyroidism.

Her Body Mass Index was $13 \mathrm{~kg} / \mathrm{m}^{2}$ and a Montreal Cognitive Assessment score of 27/30. Examination of lower limbs revealed bilaterally reduced muscle bulk, increased tone at all joints, reduced power of all the movements (grade 3/5), diminished tendon reflexes, and equivocal plantar responses. Sensory examination was normal. However Joint position sense and vibration sense were impaired. Romberg sign was positive and a scissoring type of spastic gait was observed. All the muscle groups of the right upper limb showed wasting, hypertonia, and hyperreflexia with normal sensation. Her peripheral blood revealed; white blood cell count 
$8.5 \times 109 / \mathrm{L}$, hemoglobin $12.8 \mathrm{~g} / \mathrm{dL}$, mean corpuscular volume $101 \mathrm{fL}$, and platelets $61 \times 109 / \mathrm{L}$. The blood picture showed oval macrocytes but there were no hyper segmented neutrophils. There were high signal intensities in the MRI of the dorsal columns of the cervical spinal cord suggestive of subacute combined degeneration of the cord [Figure 01]. feel better after 48 hours of treatment. There was a reticulocyte response within 3 days. Her platelet counts slowly rose from $61 \times 109$ /L to $120 \times 109$ /L over 4 weeks. She was able to walk with an improvement of limb weakness and hypertonia with physiotherapy. However, there was no objective improvement of muscle power neither her vision even after 3 months of treatment. She also developed

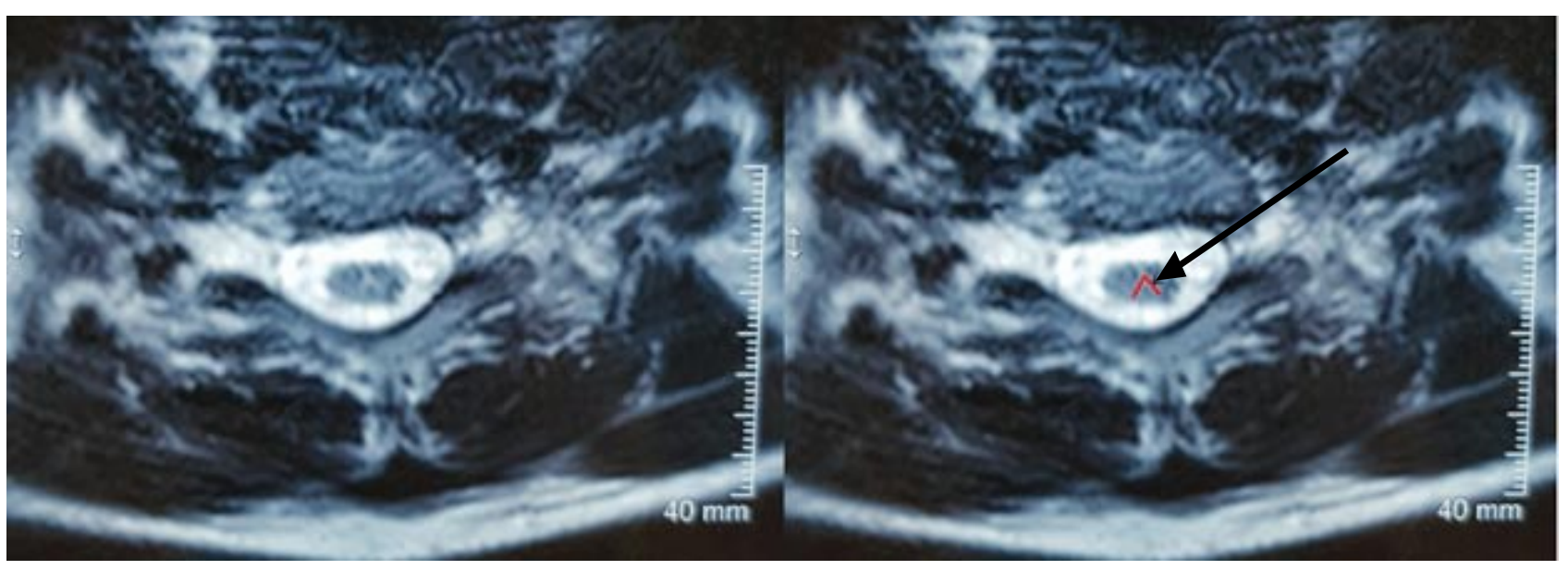

Figure 1: MRI scan of the cervical spine showing inverted " $V$ " sign (black arrow) of dorsal column high signal intensity suggestive of subacute combined degeneration

MRI brain showed atrophy of the left frontoparietal region which may be the focus for epilepsy and cause for the spastic monoplegia of the right upper limb possibly due to an insult during childhood. There was no MRI evidence of demyelination and optic neuritis.

The color optic fundoscopy revealed pallor of the temporal region of the optic discs suggestive of nutritional optic atrophy [Figure 02].
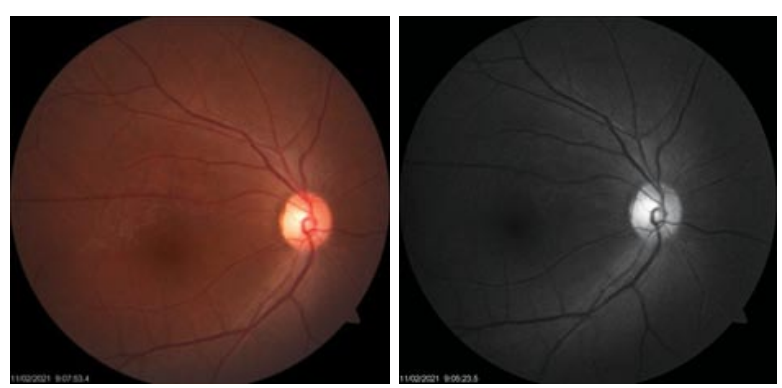

Figure 2: Color Fundus photography showing temporal pallor

Ultrasound scan of the abdomen showed normal liver and other organs.

With the clinical suspicion, she was started on Intramuscular B12 (Hydroxocobalamin) after taking bloodfor B12 and folate levels. She started to

\begin{tabular}{ll}
\hline Investigation & Results \\
\hline WBC & $8.5 \times 10^{9} / \mathrm{L}$ \\
\hline Neutrophils & $4.1 \times 10^{9} / \mathrm{L}$ \\
\hline $\mathrm{Hb}$ & $12.8 \mathrm{~g} / \mathrm{dL}$ \\
\hline $\mathrm{MCV}$ & $101 \mathrm{fL}$ \\
\hline $\mathrm{MCH}$ & $33.5 \mathrm{pg}$ \\
\hline MCHC & $34.4 \mathrm{~g} / \mathrm{dL}$ \\
\hline Platelet & $61 \times 10^{9} / \mathrm{L}$ \\
\hline ESR & $03 \mathrm{~mm} / 1^{\text {st }}$ hour \\
\hline CRP & $5 \mathrm{mg} / \mathrm{dL}$ \\
\hline AST & $24 \mathrm{IU} / \mathrm{L}$ \\
\hline ALT & $20 \mathrm{IU} / \mathrm{L}$ \\
\hline Serum sodium & $141 \mathrm{mmol} / \mathrm{L}$ \\
\hline Serum potassium & $3.4 \mathrm{mmol} / \mathrm{L}$ \\
\hline Albumin & $3.2 \mathrm{~g} / \mathrm{dL}$ \\
\hline Globulin & $2.4 \mathrm{~g} / \mathrm{dL}$ \\
\hline TSH & $1.58 \mathrm{mlU} / \mathrm{L}$ \\
\hline T4 & $1.08 \mathrm{ng} / \mathrm{dL}$ \\
\hline LDH & $142 \mathrm{IU} / \mathrm{L}$ \\
\hline VDRL & Negative \\
\hline HIV 1 and 2 antibodies & Negative \\
\hline
\end{tabular}

Table 1: Initial Laboratory investigation results 
hypokalemia 1 week after the treatment which was suggestive of erythropoiesis owing to successful treatment.

Serum B12 levels and folic acid levels were $60 \mathrm{pg} / \mathrm{mL}$ (normal $>300$ ) and $10 \mathrm{ng} / \mathrm{mL}$ (normal >4)respectively. The upper gastrointestinal endoscopic study was normal with normal gastric mucosa. Small bowel barium meal and follow-through were normal. She also had normal levels of anti-Intrinsic factor antibodies and anti-parietal cell antibodies. Serum copper and zinc levels were also normal. The nerve conduction study showed no large fiber peripheral neuropathy. Her laboratory investigations are shown in table 01.

Her hematological response to the treatment is shown in Table 2.
The predictability of hematological and clinical responses to Vitamin B12 therapy within particular timeframes is well known. ${ }^{6}$ Serum lactate dehydrogenase (LDH) and indirect hyperbilirubinemia are markers of hemolysis reduced within the first 24 to 48 hours. After 3 to 4 days the reticulocyte count increases. If there is anemia the hemoglobin levels start to increase by 1 to 2 weeks and come back to normal by 4 to 8 weeks. Other hematological manifestations such as leukopenia, thrombocytopenia, and hyper segmented neutrophils resolve within 2 to 4 weeks. The neurological manifestations may show some improvement after 3 months which may even take up to one year. ${ }^{7}$ Occasionally the neurological deficits may be irreversible particularly if left untreated for a longer period. ${ }^{7}$

\begin{tabular}{llllll}
\hline & Day 01 & Day 3 & Day 14 & Day 28 & 4 weeks \\
\hline WBC $\left(\times 10^{9} / \mathrm{L}\right)$ & 8.5 & 7.0 & 8.0 & 7.2 & 8.0 \\
$\mathrm{Hb}(\mathrm{g} / \mathrm{dL})$ & 12.8 & 12.5 & 12.2 & 12.3 & 12.5 \\
$\mathrm{MCV}(\mathrm{fL})$ & 101 & 102 & 100 & 97 & 95 \\
\hline Platelet $\left(\times 10^{9} / \mathrm{L}\right)$ & 61 & 66 & 72 & 88 & 120 \\
\hline Reticulocyte count $(\%)$ & 0.88 & 2.21 & 4.3 & 3.2 & 2.0 \\
\hline
\end{tabular}

Table 2: Improvement of hematological parameters with treatment

\section{Discussion}

Vitamin B12 deficiency is commonly found in all vegetarian groups. ${ }^{4}$ Our patient had low serum B12 levels and normal folate levels suggestive of cobalamin deficiency. She had bilateral nutritional optic atrophy, subacute combined degeneration of the spinal cord, low platelets, and macrocytosis which are well-recognized manifestations of cobalamin deficiency.

In this patient, the long-term use of sodium valproate may have contributed to the B12 deficiency.Serum B12 and folate levels are found to be low after initiation of treatment with various antiepilepticsspecially with carbamazepine and phenytoin sodium. ${ }^{5}$

Even though this patient had optic atrophy and subacute combined degeneration of the spinal cord there was no anemia. Megaloblastic anemia is a major manifestation of vitamin B12 deficiency. Our patient had macrocytosis but no anemia or hyper segmentedneutrophils which is a known entity of cobalamin deficiency with neuropsychiatric manifestations in the absence of anemia. ${ }^{2}$ Concomitant folate supplementation may have led to the masking of hematological manifestations of cobalamin deficiency may delay the presentation and will result in neurological manifestations. ${ }^{3}$

\section{Conclusion}

B12 deficiency is a known finding in vegans which is classically associated with megaloblastic anemia. However, the clinician should understand neurological manifestations may present even in the absence of anemia. In addition, folic acid supplementation may lead to worsening neurological manifestations and masking of hematological manifestations. keeping a high index of suspicion is needed because early diagnosis and treatment carries a better outcome.

\section{References}

1. Reynolds E. Lancet Neurology 2006; 5: 949-60 DOI: 10.1016/S1474-4422(06)70598-1.

2. J Lindenbaum, E B Healton, D G Savage, J C Brust, T J Garrett, E R Podell, P D Marcell, S P Stabler, R $H$ Allen. Neuropsychiatric manifestations caused by cobalamin deficiency in the absence of anemia or macrocytosis. The new England journal of medicine; 1988 Jun 30;318(26) DOI: 10.1056/ NEJM198806303182604

3. Jacob Selhub, Martha Savaria Morris, Paul F Jacques; In Vitamin B12 deficiency, higher serum folate is associated with increased total homocysteine and methylmalonic acid concentrations; Pro- 
ceedings of the national academy of sciences of the United State of America 2007 Dec 11;104(50) DOI: $10.1073 /$ pnas. 0709487104

4. Tekin Guney, Aysun Senturk Yikilmaz, Imdat Dilek. Epidemiology of vitamin B12 deficiency. Epidemiology of Communicable and non-communicable diseases- attributes of lifestyle and nature on humankind 2016; chapter 07: 103-112 DOI: $10.5772 / 63760$

5. Hong-Li Huang, Hao Zhou, Nuan Wang, Chun-Yu Yu. Effects of antiepileptic drugs on the serum folate and vitamin B12 in various epileptic patients. Biomedical Reports 201165 (4): 413-416 DOI: 10.3892/br.2016.737

6. Sally P Stabler. The new England journal of medicine 2013 Jan 10;368(2):149-60 DOI: 10.1056/ NEJMcp1113996

7. Olavo $\mathrm{M}$ Vasconcelos 1 , Erika $\mathrm{H}$ Poehm, Robert J McCarter, William W Campbell, Zenaide M N Quezado. Potential outcome factors in subacute combined degeneration: review of observational studies. Journal of general internal medicine. 2006 Oct;21(10):1063-8. DOI: 10.1111/j.15251497.2006. 00525.x 